EOMmUn: Communication et organisation

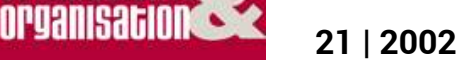

Espace et communication dans les organisations

\title{
Place et enjeux des revues pour la recherche en Infocom
}

Hugues Hotier

\section{OpenEdition \\ 12 Journals}

Édition électronique

URL : http://journals.openedition.org/communicationorganisation/2698

DOI : 10.4000/communicationorganisation.2698

ISSN : $1775-3546$

Éditeur

Presses universitaires de Bordeaux

Édition imprimée

Date de publication : 1 mai 2002

ISSN : 1168-5549

Référence électronique

Hugues Hotier, «Place et enjeux des revues pour la recherche en Infocom », Communication et organisation [En ligne], 21 | 2002, mis en ligne le 27 mars 2012, consulté le 19 avril 2019. URL : http:// journals.openedition.org/communicationorganisation/2698; DOI : 10.4000/ communicationorganisation.2698

Ce document a été généré automatiquement le 19 avril 2019

(c) Presses universitaires de Bordeaux 


\title{
Place et enjeux des revues pour la recherche en Infocom
}

\author{
Hugues Hotier
}

1 Les 25 et 26 mars, à l'invitation de la Société Française des Sciences de l'Information et de la Communication et du Laboratoire d'Anthropologie "Mémoire. Identité, Cognition sociale » que co-dirige le professeur Paul Rasse, un nombre important de chercheurs en infocom se retrouvaient à l'Université de Nice pour une réflexion sur les revues scientifiques et leur place dans la recherche. Malgré leurs faibles tirages, ces revues sont cependant très lues, ne serait-ce que parce qu'elles figurent en bonne place dans les bibliothèques universitaires. Et pourtant, comme le rappellent Daniel Bougnoux et Paul Rasse dans une récente livraison de La lettre d'Inforcom, une enquête récente menée à la demande du Ministère de la recherche par Philippe Janin est révélatrice de la sélection drastique qu'opèrent les membres de la communauté des sciences de l'information et de la communication. Sur une liste de 263 revues recensées en Infocom et disciplines proches au plan mondial, seulement 17 sont connues par un chercheur sur deux ${ }^{1}$. Sur les 40 revues françaises, 12 seulement sont connues par une personne sur deux : Cahiers de Médiologie, CinémAction, Communication et Langages, Communication \& Organisation, Communications, Études de communication, Hermès, MEI, Quaderni, Réseaux, Revue Française de Sociologie, Sciences de la Société. Comme le font remarquer les auteurs de l'article, "C'est dire la dispersion et la méconnaissance des revues censées structurer notre discipline. »

2 Pour Jean-Marc Lévy-Leblond, la revue est un lieu de validation, de diffusion et d'archivage scientifique, en particulier dans les bibliothèques, universitaires ou non. La validation est. elle, assurée par les pairs, notamment par le biais des comités de lecture. Ce modèle tend à être contrebalancé, en sciences dures particulièrement, par de nouvelles pratiques: prê-tirages envoyés par courrier à des membres repérés de la communauté, développement de la bibliométrie permettant de repérer le nombre des citations et des reprises d'articles, aggravation de la spécialisation qui entraîne un morcellement des publics... Autre nouveauté, il existe des revues qui exigent du laboratoire auquel appartient l'auteur un paiement pour la publication de son article. Il est vrai que, de plus en plus, on voit apparaître dans les revues de sciences dures une 
signature institutionnelle (le nom du laboratoire) plutôt que personnelle (le nom de l'auteur). Patrice Flichy propose une typologie qui range les revues de sciences dures en trois catégories ajoutant au modèle proposé par Jean-Marc Lévy-Leblond la revue d'idées, qui construit un modèle intellectuel cohérent, et la revue de diffusion des savoirs.

Une table ronde, qui rassemblait Daniel Bougnoux, Hugues Hotier et Yves Jeanneret, permit de traiter de questions plus centrées sur les revues de sciences humaines et sociales. Furent abordées notamment la question de la légitimation par l'existence d'un comité de lecture travaillant "à l'aveugle » comme dans les dégustations de vin et celle de la prééminence finale du comité éditorial qui assume la responsabilité de la publication d'un article et garantit la ligne éditoriale de la revue. Autre question traitée : peut-on dire qu'une revue exprime une cohérence scientifique ? Celle-ci est réelle même quand elle ne s'affiche pas explicitement. La constitution de l'équipe éditoriale autour du fondateur résulte le plus souvent d'une cooptation sur des critères de proximité scientifique.

Autre débat apprécié, celui qui traitait de la revue comme outil de la recherche permit de confronter les points de vue de Philippe Quinton, Saïda Habbab, Michaël Palmer, Geneviève Jacquinot, Emmanuel Paris, Philippe Hert et Viviane Couzinet. Les avis furent partagés sur cette question de l'articulation de la revue et de la recherche: faut-il distinguer la revue de recherche de la revue ouverte à un public plus large ? Cela équivaut à s'interroger sur l'utilité sociale de la recherche... Quand un écrit de recherche devient publication, il change de statut, il acquiert une sorte de légitimation. À ne pas confondre avec une certification car, par définition, celle-ci ne saurait être conférée que par une expertise externe et non par l'agrément d'un membre de la communauté.

5 Finalement, une revue a, certes, un projet éditorial mais c'est, in fine, l'étude du lectorat qui, seule peut dire ce qu'elle est: de recherche, de vulgarisation, professionnelle... On peut même se demander si elle ne peut avoir plusieurs statuts à la fois. Comme le faisait remarquer un participant, entre vulgarisation et science, on est plus dans le fil tendu que dans l'opposition. Certains débats suscitèrent des oppositions assez vives. Ainsi, lorsque Emmanuel Paris rendit compte de l'étude qu'il avait menée sur la revue Réseaux, qu'il qualifie de «littérature d'entreprise malgré la relative autonomie prise en 1999 par rapport à France-Télécom ", ses conclusions rencontrèrent-elles un désaccord de la part de Patrice Flichy. Emmanuel Paris observe que $88 \%$ des articles parus en 2000 sont de nature techniciste, c'est à dire centrés sur une nouvelle technique et/ou fondés sur une approche technique pour expliquer des logiques sociales. Dans cette revue, les sociologues dominent et les chercheurs en sciences de l'information et de la communication sont absents ou, à tout le moins minoritaires. D'autant que nombre d'auteurs relevant des SIC se présentent comme sociologues et « gagnent ainsi du prestige à peu de frais "...

6 Est-il besoin d'ajouter que cette rencontre avait le mérite de ne pas recourir à la langue de bois. Les échanges furent fructueux, y compris quand ils avaient pour thème « l'affaire Sokal » analysée par Nicolas Pélissier, Robert Boure, Bauduin Jurdant, Jean-Marc LévyLeblond et Yves Jeanneret.

7 À noter une initiative heureuse, les organisateurs du colloque avaient édité un annuaire des revues en Infocom avec une fiche pour chacune des 33 recensées. Bref, un colloque utile, riche et souvent passionnant. 


\section{NOTES}

1. L'enquête a été menée auprès des adhérents de la SFSIC, laquelle regroupe la quasi totalité des membres de la communauté des sciences de l'information et de la communication. 